\title{
The effects of nonsteroidal anti-inflammatory drugs in the incident and recurrent risk of hepatocellular carcinoma: a meta-analysis
}

This article was published in the following Dove Press journal:

OncoTargets and Therapy

20 September 2017

Number of times this article has been viewed

\author{
Qing Pangl,* \\ Hao Jin ${ }^{1, *}$ \\ Kai $\mathrm{Qu}^{2}, *$ \\ Zhongran Man' \\ Yong Wang' \\ Song Yang' \\ Lei Zhou' \\ Huichun Liu' \\ 'Department of Hepatobiliary \\ Surgery, The First Affiliated Hospital \\ of Bengbu Medical College, Bengbu, \\ Anhui, ${ }^{2}$ Department of Hepatobiliary \\ Surgery, The First Affiliated Hospital \\ of Xi'an Jiaotong University, Xi'an, \\ Shaanxi, China \\ *These authors contributed equally \\ to this work
}

Background: Recent studies have showed that nonsteroidal anti-inflammatory drugs (NSAIDs) could reduce the risk of several types of cancer. However, epidemiological evidence of the association between NSAIDs intake and the risk of hepatocellular carcinoma (HCC) remains controversial.

Methods: To assess the preventive benefit of NSAIDs in HCC, we simultaneously searched the databases of PubMed, EmBase, Web of Science, and Scopus and screened eligible publications.

Results: A total of twelve articles (published from 2000 to 2017) from five countries were identified by retrieval. We observed a significantly lower risk of HCC incidence among users of NSAIDs than among those who did not use NSAIDs (pooled hazard ratio [HR] value $=0.81$, 95\% confidence interval [CI]: 0.69-0.94). No evidence of publication bias was observed (Begg's test, $P=0.755$; Egger's test, $P=0.564$ ). However, when stratified according to the categories of NSAIDs, users of non-aspirin NSAIDs (HR $=0.81,95 \%$ CI: $0.70-0.94$ ), but not aspirin ( $\mathrm{HR}=0.77,95 \% \mathrm{CI}: 0.58-1.02)$, showed a statistically significant reduced HCC incidence. We also found that NSAIDs use significantly reduced the recurrent risk of HCC, with a HR value of 0.79 (95\% CI: 0.75-0.84), whereas there was no statistically significant association between NSAIDs use and HCC mortality, with a HR value 0.65 (95\% CI: 0.40-1.06).

Conclusion: Taken together, our meta-analysis demonstrates that NSAIDs significantly reduce the incident and recurrent risk of HCC.

Keywords: NSAIDs, aspirin, hepatocellular carcinoma, incidence, recurrence

\section{Introduction}

Hepatocellular carcinoma (HCC) is one of the leading causes of cancer-related death worldwide, especially in less developed countries. According to the Global Cancer Statistics of 2012, ${ }^{1}$ the yearly burden of HCC is estimated to be 782,500 new cases, resulting in 745,500 deaths. To lower the incidence and mortality of HCC, preventative drugs for HCC need to be explored.

A growing body of convincing evidence demonstrates that host systemic and local inflammation promotes tumorigenesis and tumor progression. ${ }^{2-5}$ More than $70 \%-90 \%$ cases of HCC gradually unfold on the background of chronically inflamed hepatic parenchyma and thus HCC represents one of the pro-inflammatory stimuli-related malignancies. ${ }^{6}$ In addition to the elevated risk of $\mathrm{HCC}$ incidence, there is increasing evidence supporting the adverse role of systemic inflammation in the prognosis of HCC. ${ }^{7,8}$
Correspondence: Huichun Liu; Lei Zho Department of Hepatobiliary Surgery, The First Affiliated Hospital of Bengbu Medical College, 287 Changhuai Road Bengbu, Anhui, 233000, China Tel +860552308 6l49

Email liuhcdoctor@I26.com; zidane20082003@I63.com 
The close association between inflammation and tumorigenesis forms the basis of the hypothesis that anti-inflammatory drugs may have antitumor effects. Based on this, recently considerable evidence from experimental and clinical studies indicates that nonsteroidal anti-inflammatory drugs (NSAIDs) could reduce the risk of several types of cancer, including gastric cancer, ${ }^{9}$ prostate cancer, ${ }^{10}$ colorectal cancer, ${ }^{11}$ esophageal cancer, ${ }^{12}$ breast cancer, ${ }^{13}$ bladder cancer, ${ }^{14}$ and head and neck cancers. ${ }^{15}$ The key mechanism of the protective action of NSAIDs is the inhibition of the cyclooxygenase (COX) enzymes, which could catalyze the synthesis of prostaglandins (PGs) in inflammatory processes. Taking into account that HCC is a chronic inflammationrelated disease and is characterized by high expression of $\mathrm{COX},{ }^{16}$ it is tempting to speculate that NSAIDs may reduce HCC risk. Herein, we used the approach of meta-analysis to summarize all eligible epidemiological evidence for an association between aspirin or non-aspirin NSAIDs use and the incident or recurrent risk of HCC.

\section{Materials and methods}

\section{Search strategy and selection criteria}

A systematic search through databases of PubMed, EmBase, Web of Science, and Scopus databases until April 2017 was performed by two independent investigators (PQ and JH). Our core search consisted of terms ("nonsteroidal anti-inflammatory drugs" or NSAIDs or aspirin) AND ("hepatocellular carcinoma" or "liver cancer"). Additionally, we retrieved the reference lists of the included publications and related reviews manually. Endnote X7 software (Thomson Corporation, Stamford, CT, USA) was used to analyze and manage references.

Studies that met the following predetermined selection criteria were included: 1) published as an original article in English; 2) designed as an observational study; 3) exposure was use of aspirin or non-aspirin NSAIDs; 4) outcome was the incidence, recurrence, or mortality of HCC; 5) reported hazard ratio (HR), relative risk, or odd ratio value and $95 \%$ confidence interval (CI), ${ }^{17,18}$ or provided sufficient data to assess them. We excluded the following studies: 1) those that assessed the effect of NSAIDs on the survival of patients with HCC; 2 ) those that just provided $P$-value or other conditions wherein effect size and $95 \%$ CI could not be estimated; 3) animal studies, reviews, conference abstracts, commentaries, editorials, and duplicate studies.

\section{Data abstraction}

Based on the above inclusive and exclusive criteria, two authors (PQ and JH) independently evaluated the retrieved publications for inclusion and discrepancy was resolved by discussion. Kappa concordance coefficient was then calculated to assess the agreement between the two screeners. We extracted the following data with a standardized data collection protocol: tumor location, first author, publication year, country, study period, duration of follow-up, information source, types of NSAIDs, median age of study population, number of participants, number of cases, HR value (adjusted HR value in preference) and 95\% CI, and controlled confounders. The research quality of each included study was assessed by MZ by using the modified Newcastle-Ottawa Scale (NOS) scores. The score ranged from zero to nine and $\geq 7$ points was defined as high quality. We adhered to the Preferred Reporting Items for Systematic Reviews and Meta-Analyses guidelines for reporting the current meta-analysis. All data were double checked by the first author (PQ).

\section{Statistical analysis}

We estimated the incident risk of HCC in users of any NSAIDs, aspirin, and non-aspirin NSAIDs compared with nonusers, respectively. With the eligible studies, we uniformly used a random-effects model to calculate the pooled HR value and $95 \%$ CI. Heterogeneity across studies was estimated by Q value and $I^{2}$ statistic. $I^{2}$ values of $25 \%, 50 \%$, and $75 \%$ correspond to cutoff points of low, moderate, and high degrees of heterogeneity, respectively. A study was considered to demonstrate statistically substantial heterogeneity provided $P<0.1$ in Q statistic or $I^{2}>50 \%$; otherwise, there was no significant heterogeneity. Based on the factors that might bring in potential heterogeneity, we subsequently performed subgroup analyses and meta-regression to seek the potential source of heterogeneity.

We also did influence analyses to evaluate whether any single study could markedly affect the pooled results. We compared the pooled HR values calculated by using a fixedeffects model with that calculated by using a random-effects model. Then, publication bias was determined by Begg's funnel plot and Egger's test. Finally, the Galbraith plot was studied to detect potential outliers, which might bias the results. We considered a statistically significant difference to be present if a bilateral $P$-value was $<0.05$.

\section{Results}

The flow chart of the literature retrieval and screening process is shown in Figure 1. Of the total 1,059 citations, 43 full text articles were assessed and 31 were further excluded. Thus, we finally included 12 publications in this meta-analysis. 


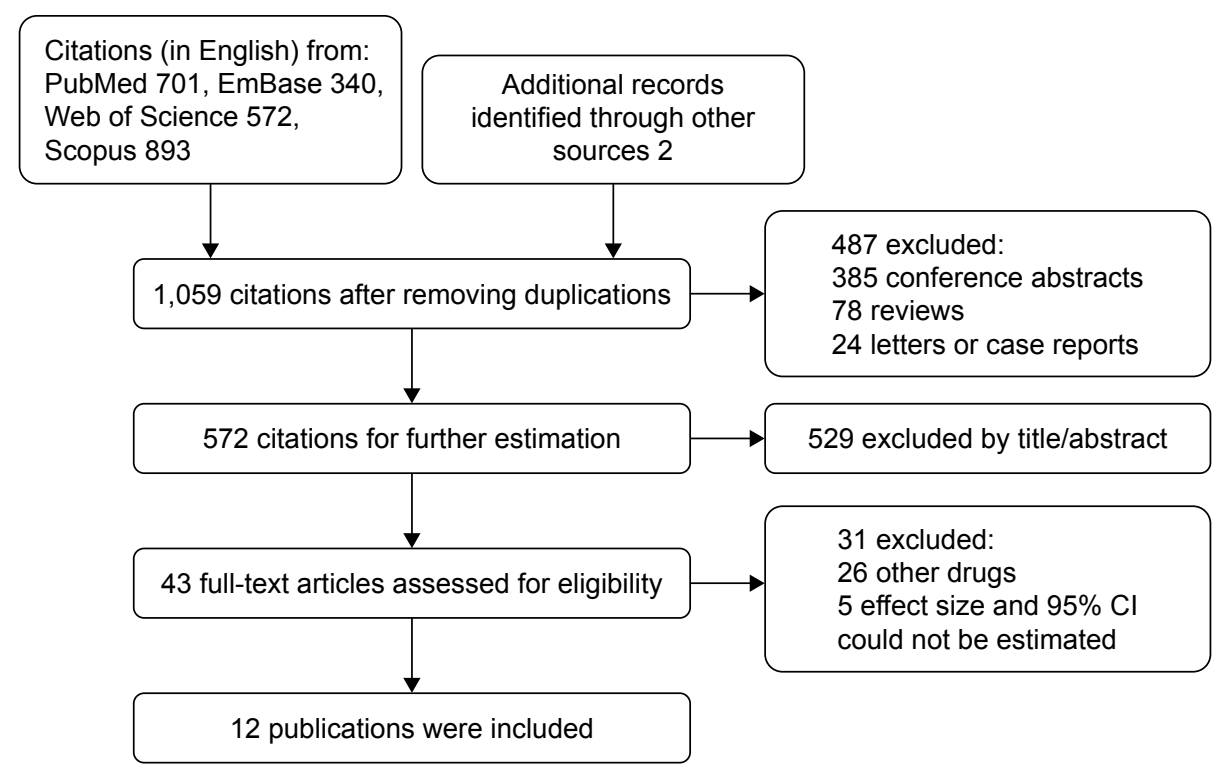

Figure I Flow chart of search strategy and study selection. Abbreviation: $\mathrm{Cl}$, confidence interval.

Agreement between the two investigators on which studies were to be included was good (kappa $=0.917$ ).

\section{Article characteristics}

The main characteristics of the twelve articles, seven of which were published after 2013, are summarized in Table 1. Five of the publications were performed in western countries and others were from Asia. Seven, four, and one were cohort, case-control, and nested case-control studies, respectively. The mean (or median) follow-up time (reported) ranged from 2.1 to 17.0 years. Nine publications controlled potential confounders and reported adjusted HR values. The assessment of study quality scores from the NOS scale demonstrated that the median score was 8 with a range from 6 to 9 , and $91.6 \%$ of the studies were high quality.

\section{Effects of NSAIDs on HCC incidence}

Seven articles with eleven studies estimated the risk of HCC incidence in individuals with NSAIDs intake compared with unexposed persons. Among five, three studies were available to estimate the associations between use of aspirin, nonaspirin NSAIDs, and risk of HCC incidence, respectively. The remaining three studies failed to distinguish aspirin from non-aspirin NSAIDs. A random-effects model was used to pool the included studies which were stratified according to the categories of NSAIDs. As shown in Figure 2A, on the whole, NSAIDs use significantly reduced the incident risk of HCC, with a HR value 0.81 (95\% CI: 0.69-0.94) and a moderate degree of between-study heterogeneity $\left(I^{2}=66.6 \%\right.$,
$P<0.001)$. The stratified analysis showed that non-aspirin NSAIDs (HR $=0.81,95 \%$ CI: $0.70-0.94$ ), but not aspirin (HR $=0.77,95 \%$ CI: $0.58-1.02)$, significantly reduced HCC incidence and was identified as potential antitumor drugs.

\section{Effects of NSAIDs on HCC recurrence and mortality}

Accumulating evidence suggests that postoperative recurrence of HCC may arise from de novo tumor in the remnant liver. By summarizing eight relevant studies with a randomeffects model, we subsequently analyzed the effects of NSAIDs on HCC recurrence. We demonstrated that NSAIDs use significantly reduced the recurrent risk of HCC, with a HR value 0.79 (95\% CI: $0.75-0.84)$ and a low degree of between-study heterogeneity (Figure $2 \mathrm{~B} ; I^{2}=0 \%, P=0.460$ ). In addition, two studies reported the association between NSAIDs and HCC mortality, and the pooled HR value was 0.65 (95\% CI: 0.40-1.06).

\section{Exploration of heterogeneity}

The meta-analysis on the association between NSAIDs and HCC incidence included eleven studies (more than ten) and produced substantial heterogeneity $(P<0.10)$. To explore the source of the heterogeneity, we subsequently performed subgroup and meta-regression analyses (Table 2). We analyzed the factors that might cause potential heterogeneity and there were no less than two studies in each subgroup. The covariates included NSAIDs categories (aspirin, non-aspirin, mixed NSAIDs), information source 


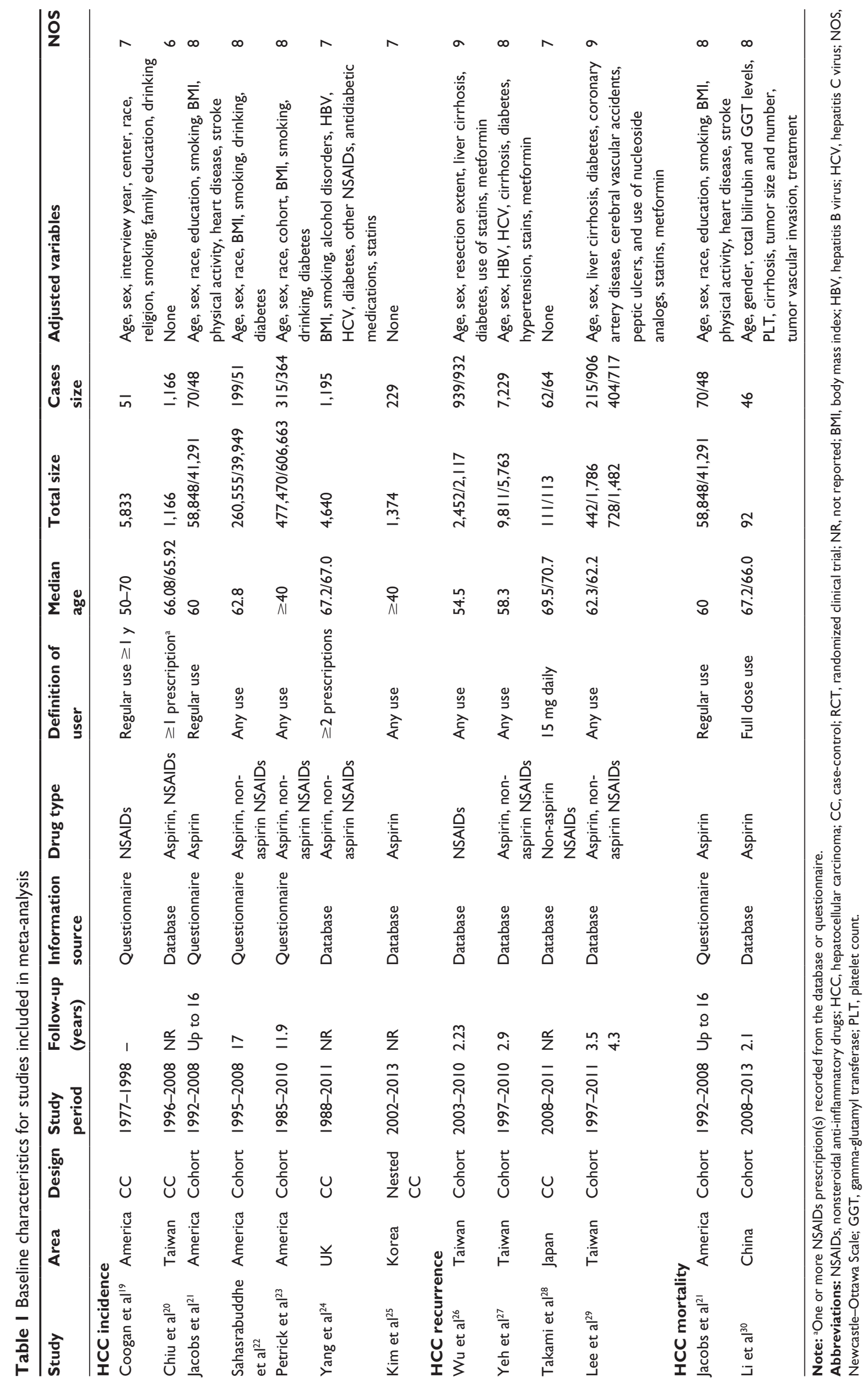




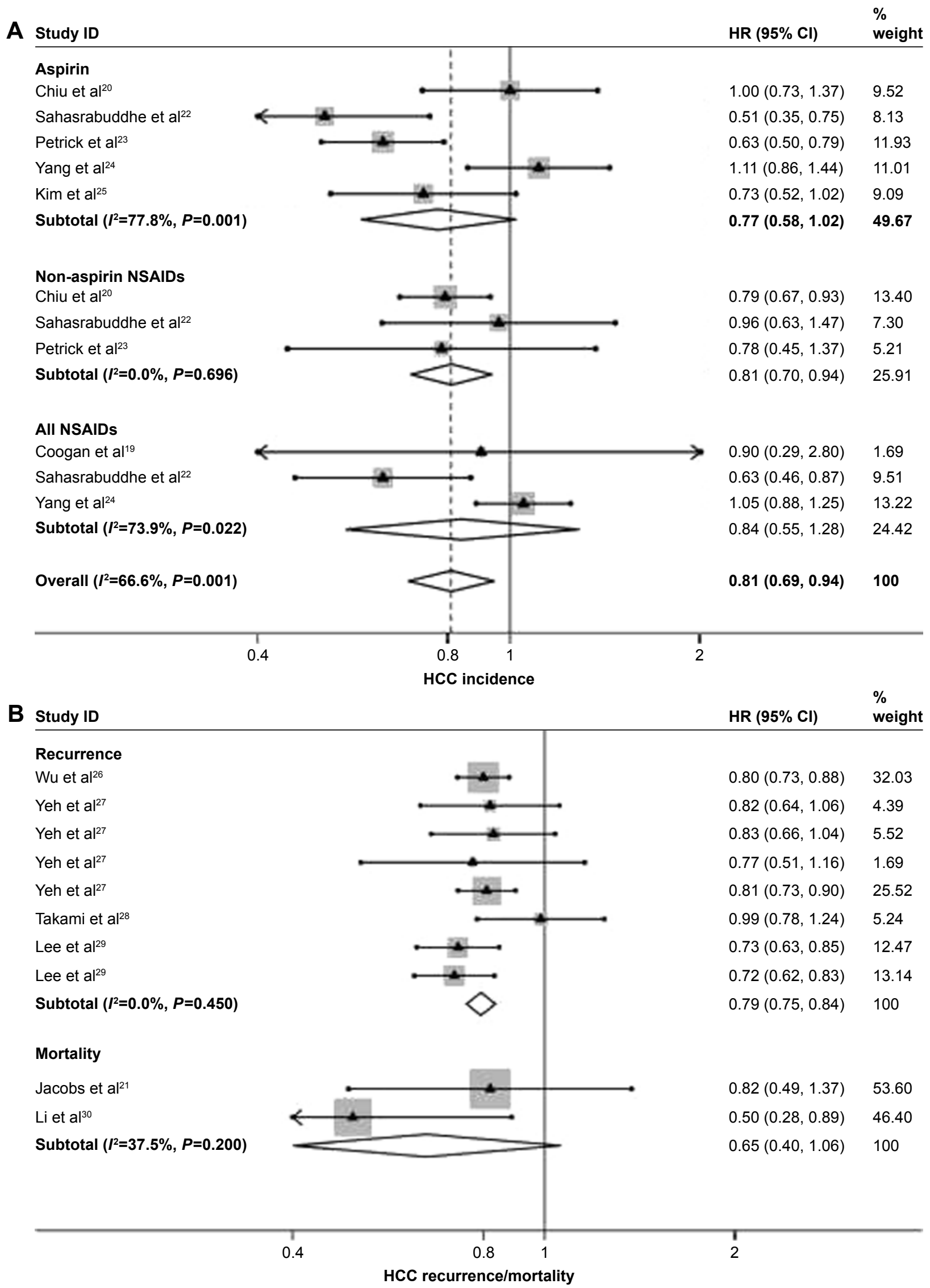

Figure 2 Forest plots of meta-analysis on the use of NSAIDs and risk of HCC incidence (A) and recurrence (B), stratified according to the categories of NSAIDs. Note: Weights are from random-effects analysis.

Abbreviations: HCC, hepatocellular carcinoma; NSAIDs, nonsteroidal anti-inflammatory drugs; $\mathrm{HR}$, hazard ratio; $\mathrm{Cl}$, confidence interval. 
Table 2 Subgroup analysis and meta-regression analysis for studies that assessed the preventive effect of NSAIDs use in HCC incident risk

\begin{tabular}{|c|c|c|c|c|c|c|c|c|c|c|}
\hline \multirow[t]{2}{*}{ Covariates } & \multirow[t]{2}{*}{ Subgroup } & \multirow{2}{*}{$\begin{array}{l}\text { No of } \\
\text { studies }\end{array}$} & \multicolumn{2}{|l|}{ HR (95\% CI) } & \multicolumn{2}{|c|}{ Heterogeneity } & \multicolumn{2}{|c|}{ Subgrouped } & \multicolumn{2}{|c|}{ Meta-regression } \\
\hline & & & $\begin{array}{l}\text { Random-effects } \\
\text { model }\end{array}$ & $\begin{array}{l}\text { Fixed-effects } \\
\text { model }\end{array}$ & $P$-value & $I^{2}$ & Q value & $P$-value & $\begin{array}{l}\text { Crude } \\
\text { P-value }\end{array}$ & $\begin{array}{l}\text { Adjusted } \\
P \text {-value }\end{array}$ \\
\hline Overall & & 11 & $0.8 \mathrm{I}(0.69-0.94)$ & $0.83(0.77-0.90)$ & 0.001 & 66.6 & & & & \\
\hline NSAIDs & Aspirin & 5 & $0.77(0.58-1.02)$ & $0.78(0.69-0.89)$ & 0.001 & 77.8 & 3.52 & 0.172 & 0.647 & - \\
\hline \multirow[t]{2}{*}{ categories } & Non-aspirin & 3 & $0.8 \mathrm{I}(0.70-0.94)$ & $0.81(0.70-0.94)$ & 0.696 & 0 & & & & \\
\hline & All NSAIDs & 3 & $0.84(0.56-1.28)$ & $0.94(0.81-1.09)$ & 0.022 & 73.9 & & & & \\
\hline Information & Database & 5 & $0.93(0.79-1.09)$ & $0.92(0.84-1.02)$ & 0.046 & 58.8 & 14.57 & $<0.001$ & 0.025 & 1.000 \\
\hline source & Questionnaire & 6 & $0.66(0.56-0.78)$ & $0.66(0.57-0.76)$ & 0.343 & 11.3 & & & & \\
\hline Publication & Before 2013 & 6 & $0.76(0.63-0.93)$ & $0.77(0.68-0.87)$ & 0.081 & 49.0 & 2.89 & 0.089 & 0.505 & - \\
\hline year & After 2013 & 5 & $0.85(0.66-1.10)$ & $0.89(0.80-0.99)$ & 0.002 & 76.8 & & & & \\
\hline \multirow[t]{2}{*}{ Region } & Asia & 3 & $0.82(0.7 I-0.94)$ & $0.8 \mathrm{I}(0.7 \mathrm{I}-0.93)$ & 0.344 & 6.30 & 0.17 & 0.685 & 0.829 & - \\
\hline & America/Europe & 8 & $0.79(0.63-1.00)$ & $0.84(0.76-0.93)$ & $<0.001$ & 74.6 & & & & \\
\hline \multirow[t]{2}{*}{ Design } & Cohort & 5 & $0.66(0.55-0.79)$ & $0.65(0.56-0.76)$ & 0.255 & 24.9 & 14.87 & $<0.001$ & 0.023 & 0.731 \\
\hline & Others & 6 & $0.93(0.80-1.08)$ & $0.92(0.84-1.02)$ & 0.084 & 48.5 & & & & \\
\hline Adjusted & No & 3 & $0.82(0.7 \mathrm{I}-0.94)$ & $0.8 \mathrm{I}(0.7 \mathrm{I}-0.93)$ & 0.344 & 6.30 & 0.17 & 0.685 & 0.829 & - \\
\hline confounders & Yes & 8 & $0.79(0.63-1.00)$ & $0.84(0.76-0.93)$ & $<0.001$ & 74.6 & & & & \\
\hline
\end{tabular}

Note: Bold values indicate statistical significance $(P<0.05)$.

Abbreviations: HCC, hepatocellular carcinoma; NSAIDs, nonsteroidal anti-inflammatory drugs; HR, hazard ratio; $\mathrm{Cl}$, confidence interval.

(database and questionnaire), publication year (before and after 2011), region (Asia and America/Europe), design (cohort and others), and whether the potential confounders were adjusted (yes or no). Among these factors, information source and study design were found to be the potential sources of heterogeneity by subgrouped analysis and univariate meta-regression $(P<0.05)$. However, no independent factor was identified when multivariable meta-regression was performed.

Then, Galbraith's plots identified four outliers as potential sources of heterogeneity (Figure S1). When the meta-analysis was further performed after excluding the four outliers, the heterogeneity nearly disappeared (Figure $\mathrm{S} 2 ; I^{2}=0 \%$, $P=0.530$ ).

\section{Sensitivity analysis and test of publication bias}

Afterwards, we carried out a sensitivity analysis to validate our findings. First, we compared the pooled HR values (that were calculated by using a random-effects model) with the estimated effect sizes by using a fixed-effects model (Table 2). There were no significant differences between the two models except that aspirin significantly reduced the risk of HCC when a fixed-effects model was used. Second, we carried out an influence analysis and the results suggested that no study could affect the summary of HCC incidence and recurrence risk estimate (Figure S3).

Eventually, we drew funnel plots for the meta-analysis of HCC incidence and the plots reflected basic symmetry
(Figure 3A and B). Furthermore, no significant publication bias was identified by Begg's test and Egger's test, with a $P$-value of 0.755 and 0.564 , respectively. In addition, there was also no publication bias in the meta-analysis of HCC recurrence (Figure 3C and D; $P$-value $=0.902$ and 0.656 , respectively).

\section{Discussion}

In the recent decades, there has been great progress in the diagnosis and treatment of HCC. However, to date, diagnosis at early stages of this malignancy is still difficult and the prognosis remains unsatisfactory. ${ }^{31}$ We need to urgently seek several effective strategies for decreasing the risk of HCC incidence and mortality.

Accumulating evidence suggests that systemic inflammatory responses play irreplaceable roles in different stages of tumor progression, such as initiation, promotion, invasion, and metastasis. ${ }^{32-34}$ Tumor could accelerate the inflammatory process, which in turn predisposes to tumor progression via inhibiting apoptosis, promoting angiogenesis and DNA damage. ${ }^{35}$ Recently, it has been reported that inflammation increases the incident risk of several types of cancer, such as colon cancer, prostate carcinoma, and pancreatic cancer, ${ }^{36-38}$ whereas the possible mechanisms remain unclear. There is no denying that, early in tumorigenesis, inflammatory cells produce numerous cytokines, reactive oxygen species, inflammatory mediators, and eventually provide an attractive environment for tumor formation and angiogenesis. ${ }^{39-41} \mathrm{COX}-1$ and $\mathrm{COX}-2$, which catalyze the 

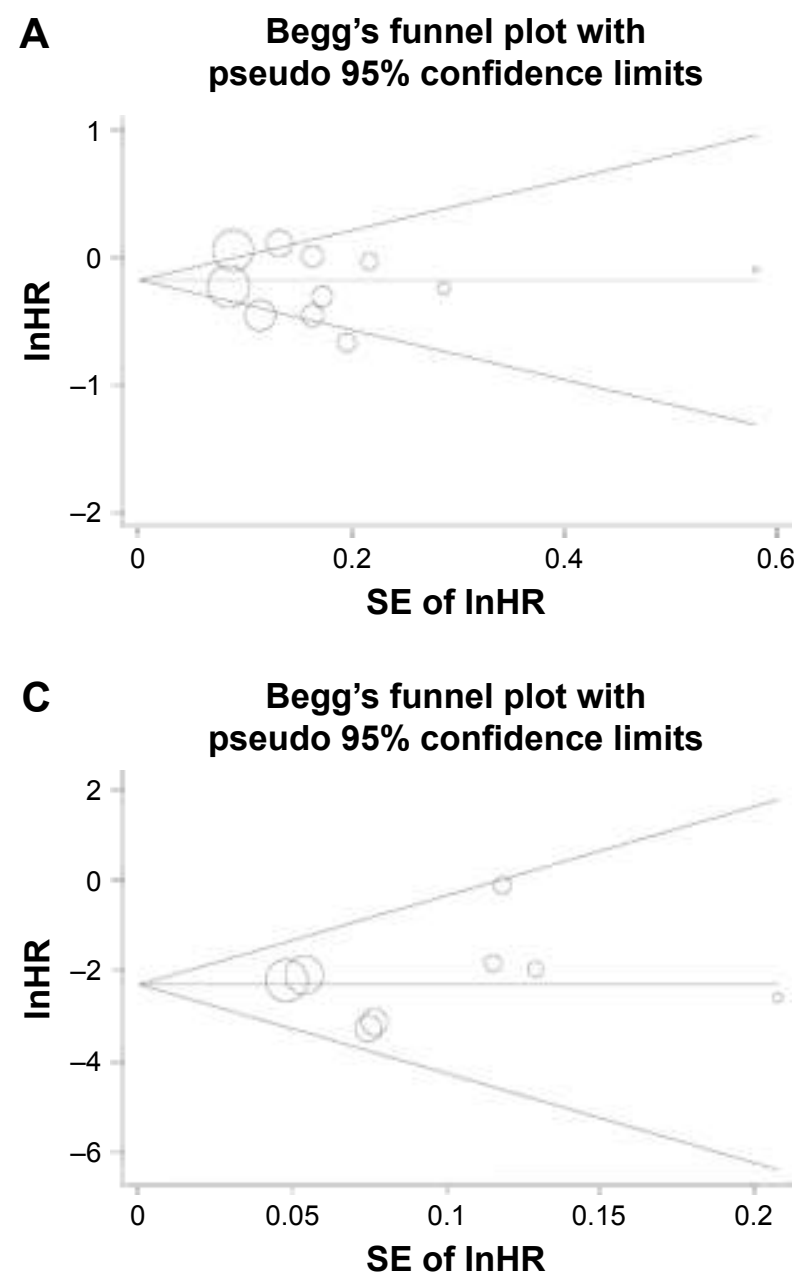
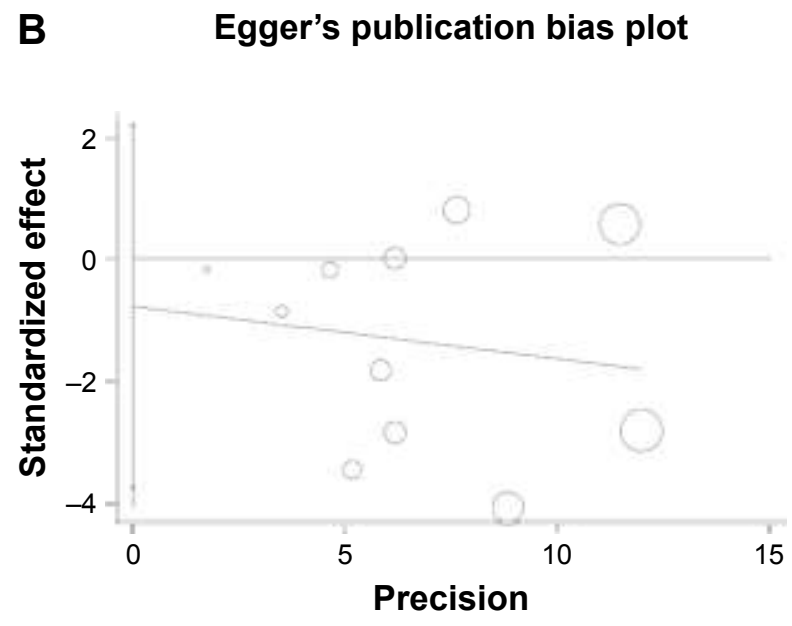

D Egger's publication bias plot

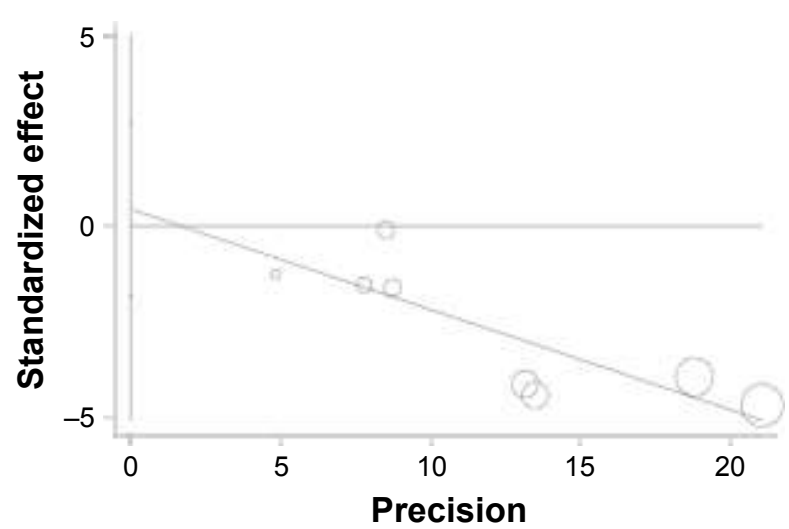

Figure 3 Begg's funnel plots and Egger's funnel plots of studies that assessed the effects of NSAIDs use in risk of HCC incidence (A, B) and recurrence (C, D). Abbreviations: HCC, hepatocellular carcinoma; NSAIDs, nonsteroidal anti-inflammatory drugs; HR, hazard ratio.

oxidative conversion of arachidonic acid to $\mathrm{PG}$, are crucial inflammatory mediators. ${ }^{42}$ By increasing PG synthesis and stimulating inflammation processes, $\mathrm{COX}-1$ and $\mathrm{COX}-2$ can promote apoptosis, angiogenesis, and tumorigenesis.

In view of the causal relationship between inflammation response and tumorigenesis, it is postulated that the development of cancer could be inhibited by alleviating inflammation. NSAIDs have been widely used for the prevention and treatment of various diseases by relieving the inflammation process. Recently, several epidemiological studies have indicated that frequent use of NSAIDs significantly reduces the incident risk of several solid cancers, such as prostate cancer, ${ }^{10}$ colorectal cancer, ${ }^{11}$ and breast cancer. ${ }^{13}$ Nevertheless, the antineoplastic mechanism of NSAIDs is not well understood and may be partially related to the inhibition of COX and PG synthesis. ${ }^{43}$ Aspirin is a strong, irreversible inhibitor of COX-1 and an inhibitor of COX-2 only at higher doses, whereas non-aspirin NSAIDs are nonselective and reversible inhibitors of both COX-1 and COX-2. ${ }^{44}$
It is notable that, as the expression of COX can be induced by various inflammatory cytokines and tumor promoters, the effects of NSAIDs may differ by tissues. ${ }^{45}$ To date, the antitumor effect of NSAIDs in HCC remains controversial.

In our study, we first hypothesized and verified the anticancer role of NSAIDs in HCC. We showed that users of NSAIDs experienced a statistically significant lower risk of HCC incidence as well as HCC recurrence than nonusers. When the analysis was stratified by the categories of NSAIDs, non-aspirin NSAIDs were found to be superior to aspirin. This difference may be due to the different pharmacological and toxic mechanisms of NSAIDs. Further basic and clinical studies are still needed to clarify the differences of antitumor effects between aspirin and other NSAIDs. In addition, we found no significant benefit of NSAIDs in reducing the risk of HCC mortality.

Potential mechanisms linking NSAIDs and reduced HCC risk might be manifold. First, the pathogenesis of HCC is strongly related to sustained inflammation. As the 
last and the most redoubtable clinical consequence of liver cirrhosis, HCC is developed mainly based on a myriad of pro-inflammatory stimuli and is triggered by well-recognized noxae such as hepatotropic viruses infection, obesity, and alcohol consumption. ${ }^{46}$ Several signaling pathways have been identified to be associated with the inflammation process of HCC, including NF- $\mathrm{B}, \mathrm{JAK}-\mathrm{STAT}$, and MAPK pathways, etc. Second, COX-2 demonstrates high expression in HCC and can promote tumor initiation, differentiation, invasion, and metastasis. ${ }^{16,47,48}$ A meta-analysis with 11 publications further indicates that high expression of COX-2 is associated with decreased survival and worse prognosis in HCC. ${ }^{49}$ In addition, it is suggested that COX-1 plays a similar role, and $\mathrm{COX}-1$ inhibitors may show potential therapeutic implications in HCC. ${ }^{50}$ Third, there is increasing evidence that platelets promote tumor growth, angiogenesis, and metastasis. ${ }^{51}$ Meanwhile, tumor patients are often accompanied by thrombocytosis and functional abnormality of platelets. Thus, the protective role of NSAIDs in HCC may be also related to the antiplatelet function.

Though widely recognized as anti-inflammatory and antitumor agents, NSAIDs still are offset by their complications of major gastrointestinal bleeding and intracranial bleeding even with low-dose usage. ${ }^{52,53}$ Moreover, bleeding risk in HCC patients treated with NSAIDs is also higher than in those not treated with NSAIDs. ${ }^{29}$ Therefore, the clinical benefit of NSAIDs in an actual clinical setting may be not satisfactory. Although NSAIDs may cause gastrointestinal bleeding and ulcer perforation, several quantitative reviews report that NSAIDs use, long-term ( $\geq 4$ years) and (or) low frequency (1-4.5 times per week) in particular, reduced the risk of gastric cancer (around 26\%-33\%). ${ }^{54-56}$

Our analysis embraces several limitations and shortcomings. First, there was substantial heterogeneity in our meta-analysis. In fact, owing to many potential confounders such as population characteristics, year of publication, follow-up time, and so forth, between-study heterogeneity was inevitable in any meta-analysis. By meta-regression analysis, we identified the potential source of heterogeneity: information source and study design. NSAIDs showed better antitumor effects in the subgroup of information source from questionnaire and in the subgroup of cohort study, both of which provide better evidence compared with matched subgroups. To reduce potential confounders and obtain more robust evidence, a generalized worldwide guideline for conducting observational studies of NSAIDs in HCC is needed. Second, the association of NSAIDs and aspirin with HCC risk may be related to the dose, duration, and frequency of these medicines. However, few included studies reported the effects of different dose or duration of NSAIDs in HCC risk. Further study should focus on the dose-response relationship between NSAIDs and the risk of HCC. Third, when a fixed-effects model was used, aspirin significantly reduced the risk of $\mathrm{HCC}$, unlike the result obtained from the random-effects model. This may due to the high heterogeneity among the included studies. The significance of aspirin in HCC risk should be further verified through larger, multicentric prospective studies or randomized clinical trials.

In conclusion, our meta-analysis showed that use of NSAIDs significantly reduced the incident and recurrent risk of HCC, but had no effect on HCC mortality. Our results provide significant clinical value in $\mathrm{HCC}$ prevention.

\section{Acknowledgment}

This work was supported by the National Natural Science Foundation of China (no 81600452).

\section{Disclosure}

The authors report no conflicts of interest in this work.

\section{References}

1. Torre LA, Bray F, Siegel RL, Ferlay J, Lortet-Tieulent J, Jemal A. Global cancer statistics, 2012. CA Cancer J Clin. 2015;65(2):87-108.

2. Elinav E, Nowarski R, Thaiss CA, Hu B, Jin C, Flavell RA. Inflammation-induced cancer: crosstalk between tumours, immune cells and microorganisms. Nat Rev Cancer. 2013;13(11):759-771.

3. Mantovani A, Allavena P, Sica A, Balkwill F. Cancer-related inflammation. Nature. 2008;454(7203):436-444.

4. Demaria S, Pikarsky E, Karin M, et al. Cancer and inflammation: promise for biologic therapy. J Immunother. 2010;33(4):335-351.

5. Gambhir S, Vyas D, Hollis M, Aekka A, Vyas A. Nuclear factor kappa $\mathrm{B}$ role in inflammation associated gastrointestinal malignancies. World J Gastroenterol. 2015;21(11):3174-3183.

6. Schutte K, Bornschein J, Malfertheiner P. Hepatocellular carcinomaepidemiological trends and risk factors. Dig Dis. 2009;27(2):80-92.

7. Roxburgh CS, McMillan DC. Role of systemic inflammatory response in predicting survival in patients with primary operable cancer. Future Oncol. 2010;6(1):149-163.

8. Pinato DJ, Stebbing J, Ishizuka M, et al. A novel and validated prognostic index in hepatocellular carcinoma: the inflammation based index (IBI). J Hepatol. 2012;57(5):1013-1020.

9. Tian W, Zhao Y, Liu S, Li X. Meta-analysis on the relationship between nonsteroidal anti-inflammatory drug use and gastric cancer. Eur J Cancer Prev. 2010;19(4):288-298.

10. Wang X, Lin YW, Wu J, et al. Meta-analysis of nonsteroidal antiinflammatory drug intake and prostate cancer risk. World J Surg Oncol. 2014;12:304.

11. Andersen V, Vogel U. Systematic review: interactions between aspirin, and other nonsteroidal anti-inflammatory drugs, and polymorphisms in relation to colorectal cancer. Aliment Pharmacol Ther. 2014;40(2): $147-159$.

12. Corley DA, Kerlikowske K, Verma R, Buffler P. Protective association of aspirin/NSAIDs and esophageal cancer: a systematic review and meta-analysis. Gastroenterology. 2003;124(1):47-56. 
13. Takkouche B, Regueira-Mendez C, Etminan M. Breast cancer and use of nonsteroidal anti-inflammatory drugs: a meta-analysis. J Natl Cancer Inst. 2008;100(20):1439-1447.

14. Zhang H, Jiang D, Li X. Use of nonsteroidal anti-inflammatory drugs and bladder cancer risk: a meta-analysis of epidemiologic studies. PLoS One. 2013;8(7):e70008.

15. Tang L, Hu H, Liu H, Jian C, Wang H, Huang J. Association of nonsteroidal anti-inflammatory drugs and aspirin use and the risk of head and neck cancers: a meta-analysis of observational studies. Oncotarget. 2016;7(40):65196-65207.

16. Guo Z, Jiang JH, Zhang J, et al. COX-2 promotes migration and invasion by the side population of cancer stem cell-like hepatocellular carcinoma cells. Medicine (Baltimore). 2015;94(44):e1806.

17. Greenland S. Quantitative methods in the review of epidemiologic literature. Epidemiol Rev. 1987;9:1-30.

18. Liu F, Yan L, Wang Z, et al. Metformin therapy and risk of colorectal adenomas and colorectal cancer in type 2 diabetes mellitus patients: a systematic review and meta-analysis. Oncotarget. 2017;8(9):16017-16026.

19. Coogan PF, Rosenberg L, Palmer JR, et al. Nonsteroidal anti-inflammatory drugs and risk of digestive cancers at sites other than the large bowel Cancer Epidemiol Biomarkers Prev. 2000;9(1):119-123.

20. Chiu HF, Ho SC, Chen CC, Yang CY. Statin use and the risk of liver cancer: a population-based case-control study. Am J Gastroenterol. 2011;106(5):894-898

21. Jacobs EJ, Newton CC, Gapstur SM, Thun MJ. Daily aspirin use and cancer mortality in a large US cohort. J Natl Cancer Inst. 2012; 104(16):1208-1217.

22. Sahasrabuddhe VV, Gunja MZ, Graubard BI, et al. Nonsteroidal anti-inflammatory drug use, chronic liver disease, and hepatocellular carcinoma. J Natl Cancer Inst. 2012;104(23):1808-1814.

23. Petrick JL, Sahasrabuddhe VV, Chan AT, et al. NSAID use and risk of hepatocellular carcinoma and intrahepatic cholangiocarcinoma: the liver cancer pooling project. Cancer Prev Res (Phila). 2015;8(12): 1156-1162.

24. Yang B, Petrick JL, Chen J, et al. Associations of NSAID and paracetamol use with risk of primary liver cancer in the Clinical Practice Research Datalink. Cancer Epidemiol. 2016;43:105-111.

25. Kim G, Jang SY, Han E, et al. Effect of statin on hepatocellular carcinoma in patients with type 2 diabetes: a nationwide nested casecontrol study. Int J Cancer. 2017;140(4):798-806.

26. Wu CY, Chen YJ, Ho HJ, et al. Association between nucleoside analogues and risk of hepatitis B virus-related hepatocellular carcinoma recurrence following liver resection. JAMA. 2012;308(18):1906-1914.

27. Yeh CC, Lin JT, Jeng LB, et al. Nonsteroidal anti-inflammatory drugs are associated with reduced risk of early hepatocellular carcinoma recurrence after curative liver resection: a nationwide cohort study. Ann Surg. 2015;261(3):521-526.

28. Takami Y, Eguchi S, Tateishi M, et al. A randomised controlled trial of meloxicam, a Cox-2 inhibitor, to prevent hepatocellular carcinoma recurrence after initial curative treatment. Hepatol Int. 2016;10(5): 799-806.

29. Lee PC, Yeh CM, Hu YW, et al. Antiplatelet therapy is associated with a better prognosis for patients with hepatitis B virus-related hepatocellular carcinoma after liver resection. Ann Surg Oncol. 2016; 23(Suppl 5):874-883.

30. Li JH, Wang Y, Xie XY, et al. Aspirin in combination with TACE in treatment of unresectable HCC: a matched-pairs analysis. Am J Cancer Res. 2016;6(9):2109-2116.

31. Su C. Hepatobiliary cancer: all efforts for one goal. Cancer Lett. 2016; 379(2):164-165.

32. Coussens LM, Werb Z. Inflammation and cancer. Nature. 2002; 420(6917):860-867.

33. Grivennikov SI, Greten FR, Karin M. Immunity, inflammation, and cancer. Cell. 2010;140(6):883-899.

34. Laine A, Iyengar P, Pandita TK. The role of inflammatory pathways in cancer-associated cachexia and radiation resistance. Mol Cancer Res. 2013;11(9):967-972.
35. Balkwill F, Mantovani A. Inflammation and cancer: back to Virchow? Lancet. 2001;357(9255):539-545.

36. Allin KH, Bojesen SE, Nordestgaard BG. Inflammatory biomarkers and risk of cancer in 84,000 individuals from the general population. Int $J$ Cancer. 2016;139(7):1493-1500.

37. Aleksandrova K, Boeing H, Nothlings U, et al. Inflammatory and metabolic biomarkers and risk of liver and biliary tract cancer. Hepatology. 2014;60(3):858-871.

38. Cervello M, Montalto G. Cyclooxygenases in hepatocellular carcinoma. World J Gastroenterol. 2006;12(32):5113-5121.

39. Coffelt SB, de Visser KE. Cancer: inflammation lights the way to metastasis. Nature. 2014;507(7490):48-49.

40. Ramakrishna G, Rastogi A, Trehanpati N, Sen B, Khosla R, Sarin SK From cirrhosis to hepatocellular carcinoma: new molecular insights on inflammation and cellular senescence. Liver Cancer. 2013;2(3-4): 367-383.

41. Subramaniam A, Shanmugam MK, Perumal E, et al. Potential role of signal transducer and activator of transcription (STAT) 3 signaling pathway in inflammation, survival, proliferation and invasion of hepatocellular carcinoma. Biochim Biophys Acta. 2013;1835(1):46-60.

42. Smith WL, Garavito RM, DeWitt DL. Prostaglandin endoperoxide H synthases (cyclooxygenases)-1 and -2. J Biol Chem. 1996;271(52): 33157-33160.

43. Chan AT, Ogino S, Fuchs CS. Aspirin and the risk of colorectal cancer in relation to the expression of COX-2. N Engl J Med. 2007; 356(21):2131-2142

44. Sciulli MG, Seta F, Tacconelli S, et al. Effects of acetaminophen on constitutive and inducible prostanoid biosynthesis in human blood cells. Br J Pharmacol. 2003;138(4):634-641.

45. Nagao M, Sato Y, Yamauchi A. A meta-analysis of PTGS1 and PTGS2 polymorphisms and NSAID intake on the risk of developing cancer. PLoS One. 2013;8(8):e71126.

46. Chalasani N, Horlander JC Sr, Said A, et al. Screening for hepatocellular carcinoma in patients with advanced cirrhosis. Am J Gastroenterol. 1999;94(10):2988-2993.

47. Breinig M, Rieker R, Eiteneuer E, et al. Differential expression of E-prostanoid receptors in human hepatocellular carcinoma. Int J Cancer. 2008;122(3):547-557.

48. Yang HJ, Jiang JH, Yang YT, et al. Cyclooxygenase-2 expression is associated with initiation of hepatocellular carcinoma, while prostaglandin receptor-1 expression predicts survival. World J Gastroenterol. 2016;22(39):8798-8805.

49. Chen G, Li X, Yang J, et al. Prognostic significance of cyclooxygenase-2 expression in patients with hepatocellular carcinoma: a meta-analysis. Arch Med Sci. 2016;12(5):1110-1117.

50. Lampiasi N, Fodera D, D'Alessandro N, et al. The selective cyclooxygenase-1 inhibitor SC-560 suppresses cell proliferation and induces apoptosis in human hepatocellular carcinoma cells. Int J Mol Med. 2006; 17(2):245-252.

51. Goubran HA, Stakiw J, Radosevic M, Burnouf T. Platelet-cancer interactions. Semin Thromb Hemost. 2014;40(3):296-305.

52. Jaben EA, Mulay SB, Stubbs JR. Reversing the effects of antiplatelet agents in the setting of intracranial hemorrhage: a look at the literature. J Intensive Care Med. 2015;30(1):3-7.

53. Cryer B. Reducing the risks of gastrointestinal bleeding with antiplatelet therapies. N Engl J Med. 2005;352(3):287-289.

54. Algra AM, Rothwell PM. Effects of regular aspirin on long-term cancer incidence and metastasis: a systematic comparison of evidence from observational studies versus randomised trials. Lancet Oncol. 2012; 13(5):518-527.

55. Abnet CC, Freedman ND, Kamangar F, Leitzmann MF, Hollenbeck AR, Schatzkin A. Non-steroidal anti-inflammatory drugs and risk of gastric and oesophageal adenocarcinomas: results from a cohort study and a meta-analysis. Br J Cancer. 2009;100(3):551-557.

56. Ye X, Fu J, Yang Y, Gao Y, Liu L, Chen S. Frequency-risk and durationrisk relationships between aspirin use and gastric cancer: a systematic review and meta-analysis. PLoS One. 2013;8(7):e71522. 


\section{Supplementary materials}

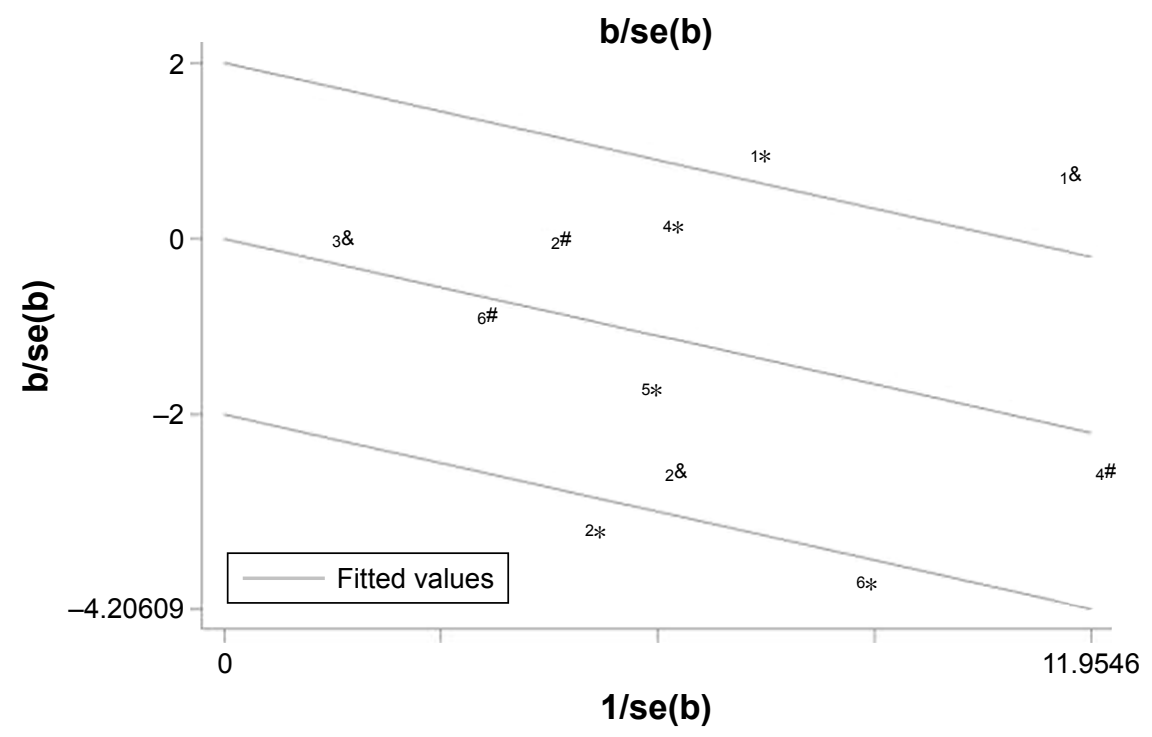

Figure SI Galbraith's plot of studies that assessed the preventive effect of NSAIDs use in risk of HCC incidence.

Notes: *Aspirin; "non-aspirin NSAIDs; *all NSAIDs.

Abbreviations: HCC, hepatocellular carcinoma; NSAIDs, nonsteroidal anti-inflammatory drugs.

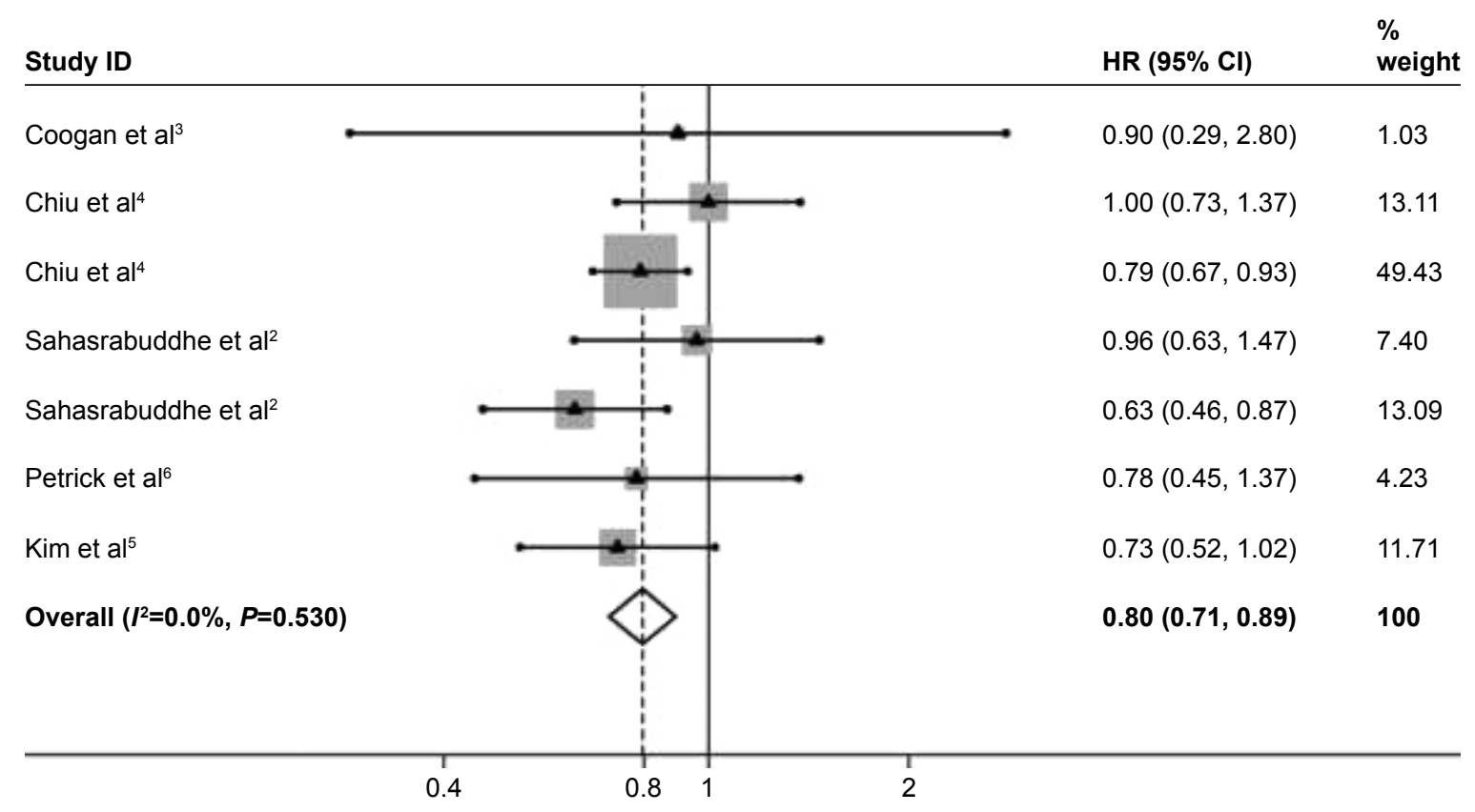

Figure S2 Forest plots of the meta-analysis on the association between NSAIDs and risk of HCC incidence after removal of the outliers of the heterogeneity. Note: Weights are from random-effects analysis.

Abbreviations: HCC, hepatocellular carcinoma; NSAIDs, nonsteroidal anti-inflammatory drugs; HR, hazard ratio; $\mathrm{Cl}$, confidence interval. 
A

Meta-analysis estimates, given named study is omitted

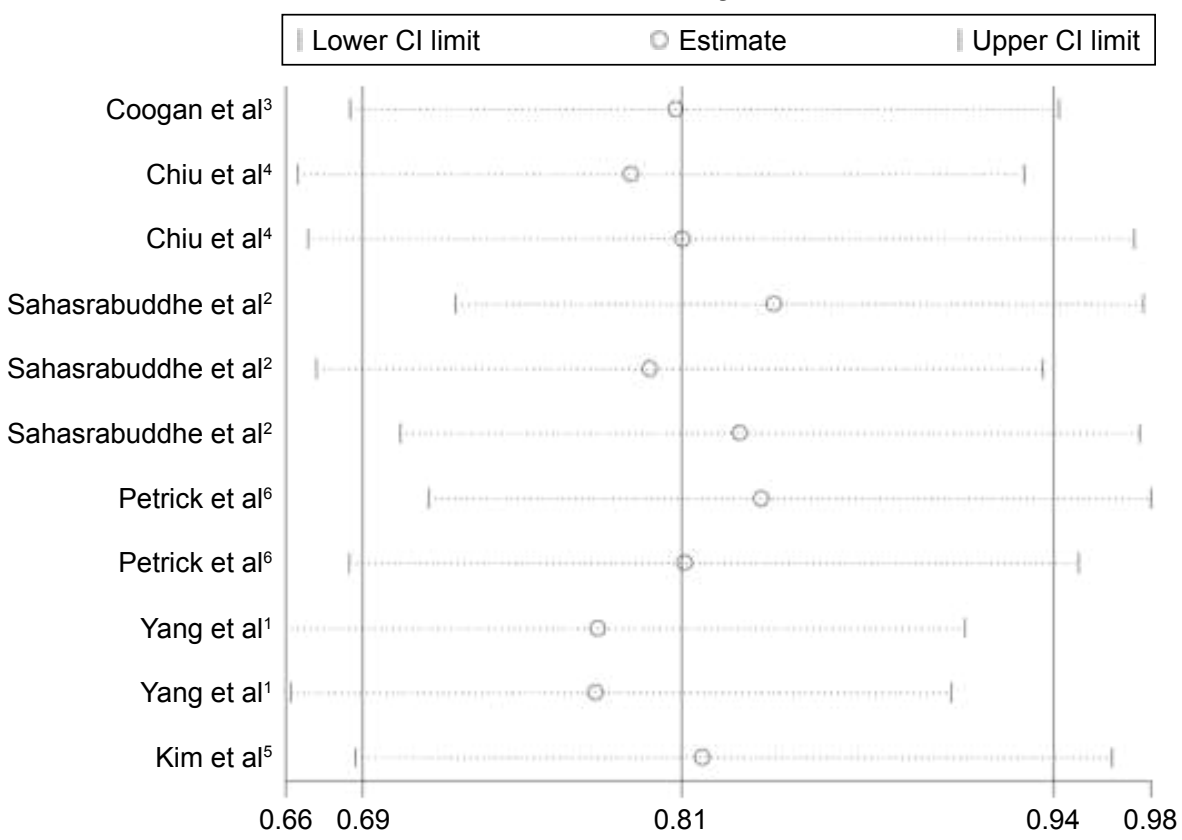

B

Meta-analysis estimates, given named study is omitted

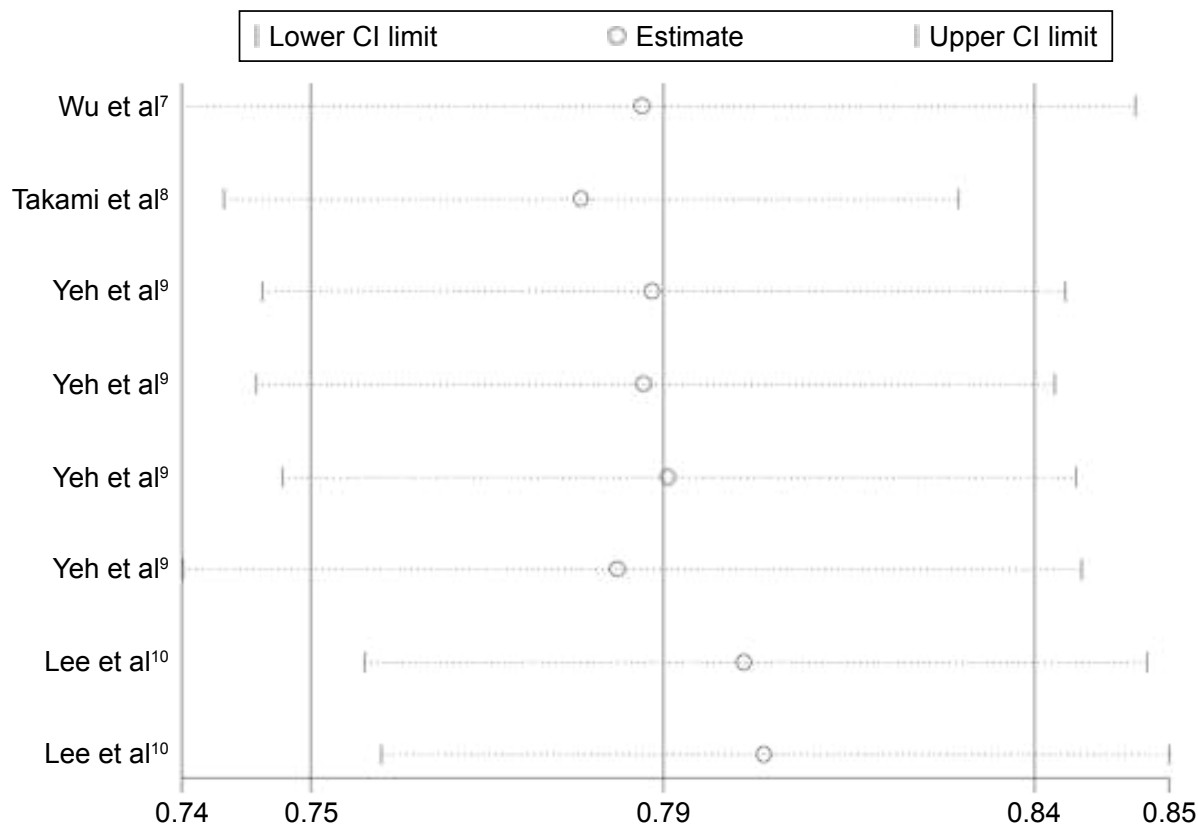

Figure S3 Influence analysis of studies that assessed the effects of NSAIDs use in the risk of HCC incidence (A) and recurrence (B).

Abbreviations: HCC, hepatocellular carcinoma; NSAIDs, nonsteroidal anti-inflammatory drugs; $\mathrm{Cl}$, confidence interval. 


\section{References}

1. Yang B, Petrick JL, Chen J, et al. Associations of NSAID and paracetamol use with risk of primary liver cancer in the Clinical Practice Research Datalink. Cancer Epidemiol. 2016;43:105-111.

2. Sahasrabuddhe VV, Gunja MZ, Graubard BI, et al. Nonsteroidal antiinflammatory drug use, chronic liver disease, and hepatocellular carcinoma. J Natl Cancer Inst. 2012;104(23):1808-1814.

3. Coogan PF, Rosenberg L, Palmer JR, et al. Nonsteroidal anti-inflammatory drugs and risk of digestive cancers at sites other than the large bowel. Cancer Epidemiol Biomarkers Prev. 2000;9(1):119-123.

4. Chiu HF, Ho SC, Chen CC, Yang CY. Statin use and the risk of liver cancer: a population-based case-control study. Am J Gastroenterol. 2011; 106(5):894-898.

5. Kim G, Jang SY, Han E, et al. Effect of statin on hepatocellular carcinoma in patients with type 2 diabetes: a nationwide nested case-control study. Int J Cancer. 2017;140(4):798-806.

6. Petrick JL, Sahasrabuddhe VV, Chan AT, et al. NSAID use and risk of hepatocellular carcinoma and intrahepatic cholangiocarcinoma: the liver cancer pooling project. Cancer Prev Res (Phila). 2015;8(12): 1156-1162.
7. Wu CY, Chen YJ, Ho HJ, et al. Association between nucleoside analogues and risk of hepatitis $\mathrm{B}$ virus-related hepatocellular carcinoma recurrence following liver resection. JAMA. 2012;308(18):1906-1914.

8. Takami Y, Eguchi S, Tateishi M, et al. A randomised controlled trial of meloxicam, a Cox-2 inhibitor, to prevent hepatocellular carcinoma recurrence after initial curative treatment. Hepatol Int. 2016;10(5): 799-806.

9. Yeh CC, Lin JT, Jeng LB, et al. Nonsteroidal anti-inflammatory drugs are associated with reduced risk of early hepatocellular carcinoma recurrence after curative liver resection: a nationwide cohort study. Ann Surg. 2015;261(3):521-526.

10. Lee PC, Yeh CM, Hu YW, et al. Antiplatelet therapy is associated with a better prognosis for patients with hepatitis B virus-related hepatocellular carcinoma after liver resection. Ann Surg Oncol. 2016; 23(Suppl 5):874-883.
OncoTargets and Therapy

\section{Publish your work in this journal}

OncoTargets and Therapy is an international, peer-reviewed, open access journal focusing on the pathological basis of all cancers, potential targets for therapy and treatment protocols employed to improve the management of cancer patients. The journal also focuses on the impact of management programs and new therapeutic agents and protocols on

\section{Dovepress}

patient perspectives such as quality of life, adherence and satisfaction The manuscript management system is completely online and includes a very quick and fair peer-review system, which is all easy to use. Visit http://www.dovepress.com/testimonials.php to read real quotes from published authors. 